\title{
CONTRIBUTION TO THE BACKGROUND RATE OF A SATELLITE X-RAY DETECTOR BY SPALLATION PRODUCTS \\ IN A CAESIUM IODIDE CRYSTAL
}

\author{
C. S. DYER and G. E. MORFILL \\ Space Physics Group, Imperial College of Science and Technology, London, S.W.7, England
}

(Received 4 June, 1971)

\begin{abstract}
The energy spectra observed in a CsI crystal in the $20 \mathrm{keV}-2 \mathrm{MeV}$ range, due to the decay of radioactive isotopes produced in the crystal by bombardment with $155 \mathrm{MeV}$ protons, are presented as a function of time after irradiation.

It is shown that the large number of decay products produced by spallation can account for these spectra and that these spectra are in quantitative agreement with the predictions of a semi-empirical formula due to Rudstam, which gives the numbers of different isotopes produced. This formula is used to predict the spallation that would occur in such a crystal on board a satellite due to cosmic rays and passages through the South Atlantic Anomaly. Inspection shows that the spallation produced in the latter case is well approximated by that at $155 \mathrm{MeV}$. Hence the experimental results are used to explain previously observed background rates and to predict the background rates that would occur in the U.K.5 X-ray telescope of Imperial College. Using the Rudstam formula an estimate of cosmic ray induced background is also made.

The relative importance of activity resulting from neutron interactions (atmospheric albedo and spacecraft secondaries) is considered.

It is suggested that the Rudstam formula provides a general method of predicting induced radioactivity in satellite materials and that observed breaks in the diffuse cosmic X-ray spectrum could be due to inadequate allowance for this source of background.
\end{abstract}

\section{Introduction}

The investigation to be described has been undertaken in order to estimate the background produced by induced radioactivity in the U.K. 5 X-ray telescope developed at Imperial College, London, for launch in 1973. The detector is a cylinder of CsI scintillator surrounded by an active anti-coincidence collimator of the same material (Figure 1). Charged particle counts are eliminated by a plastic scintillator window, which is optically coupled to the collimator. Since this type of detector is frequently used, the results presented here are of general interest.

Significant radioactive background has previously been reported by various observers using satellite-borne detectors similar to that of U.K. 5, e.g. Peterson (1965), Schwartz (1969). These investigators found that certain parts of the satellite orbit were unusable due to the contamination. Attempts have been made to explain the observed decay of activity produced in passages through the South Atlantic Anomaly in terms of the decay of one or two radioactive isotopes which can be produced in the scintillator material by neutron capture (e.g. $\mathrm{I}^{128}$ and $\mathrm{Na}^{24}$ ). It was assumed that these neutrons are produced throughout the satellite by proton bombardment, but the problem of reducing the neutron velocities to that of thermal energies has not been considered. Decay of these isotopes produces events which appear like X-rays in the 


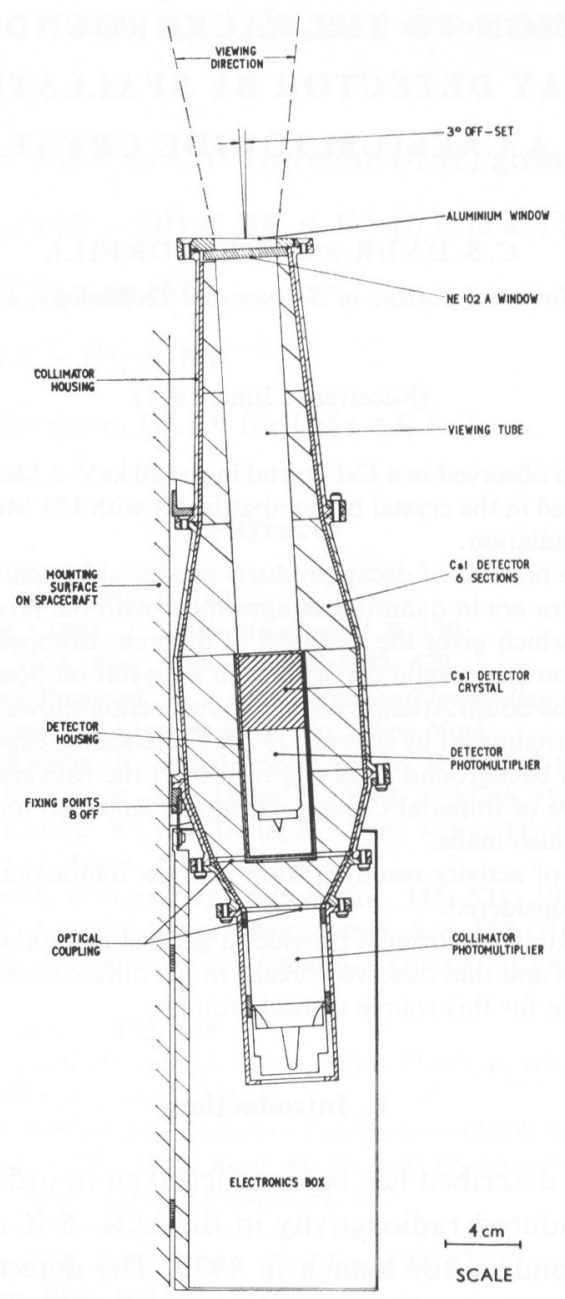

Fig. 1. The U.K.5 X-ray telescope, developed at Imperial College, London.

detector or add to the dead-time of the collimator. Recent reports have shown the induced activity to be a more complex superposition of various half-lives (Dennis, 1970). Because of the low counting rates, poor statistics and data gaps of satellite detectors, insufficient information is available to understand fully the origin of the background radiation.

The importance of the radioactive decay of spallation products resulting from inner belt proton bombardment as a source of background in satellite-borne G-M counters was pointed out by Elliot (1965).

\section{Theoretical Prediction of Nuclide Production by Spallation}

Rudstam (1966) has derived a five parameter formula which gives the production 
cross-section $\sigma\left(A_{t}, E, Z, A\right)$ for a given nucleus $(Z, A)$ when a target nucleus $\left(Z_{t}, A_{t}\right)$ is bombarded by protons of energy $E(\mathrm{MeV})$

$$
\begin{aligned}
\sigma\left(A_{t}, E, Z, A\right)= & \sigma_{i}\left(A_{t}\right) \times \\
& \times\left\{\frac{d^{2 / 3} A_{t}^{2 e / 3} \exp \left[P A-R\left(Z-S A+V A^{2}\right)^{3 / 2}\right]}{1.79\left[\exp \left(P A_{t}\right)\left(1-\frac{2 e}{3 P A_{t}}\right)-1+\frac{2}{3} e\left(1+\frac{1}{P A_{t}}\right)\right]}\right\} \times \\
& \times \exp \left(0.0074 A_{t}-0.25\right) \times f(E)
\end{aligned}
$$

where

$$
\begin{aligned}
P & =20 E^{-0.77} \text { for } E<2.1 \mathrm{GeV} \\
P & =0.056 \text { for } E \geqslant 2.1 \mathrm{GeV} \\
R & =11.8 A^{-0.45} \\
S & =0.486 \\
V & =3.8 \times 10^{-4} \\
d & =11.8 \\
e & =0.45 \\
f(E) & =\exp (1.73-0.0071 E) \text { for } E<240 \mathrm{MeV} \\
f(E) & =1 \quad \text { for } E \geqslant 240 \mathrm{MeV} \\
\sigma_{i}\left(A_{t}\right) & =\pi\left(1.26 \times 10^{-13}\right)^{2} A_{t}^{2 / 3} \mathrm{~cm}^{2}
\end{aligned}
$$

Rudstam demonstrated the validity of the formula to within an overall factor of 2 or 3 for proton energies from $50 \mathrm{MeV}$ to $30 \mathrm{GeV}$ and for targets from ${ }^{23} \mathrm{~V}$ to ${ }^{83} \mathrm{Bi}$. Relative values for the different isotopes produced from a given target may be more accurate.

Funk and Rowe (1967) have shown that the formula can be in error by as much as a factor 60 in isolated cases for products with atomic numbers near that of the target. Waddington (1969) has taken the cross-section for single nucleon removal to be $0.08 \sigma_{i}$ (where $\sigma_{i}$ is the total inelastic interaction cross-section), and has considered neutron or proton loss to be equally probable. Hence the normalisation

is applied.

$$
\sum_{Z, A} \sigma\left(A_{t}, Z, A, E\right) \leqslant 0.92 \sigma_{i}\left(A_{t}\right)
$$

These assumptions are used in this work.

The formula also gives a considerable underestimate of the numbers of light fragments, as these are produced as evaporation products rather than residual nuclei.

The inelastic interaction cross-section $\left(\sigma_{i}\right)$ was taken as 1260 millibarns for both $\mathrm{Cs}$ and $\mathrm{I}$, giving an interaction length $(\lambda)$ of $38 \mathrm{~cm}$. The formula was used to predict the quantities of isotopes produced in the central crystal of the U.K.5 detector for three different cases.

\section{A. INNER BELT PRODUCED}

The omnidirectional differential energy spectrum in the South Atlantic Anomaly was taken to have a peak flux

$$
j(E)=2 \times 10^{6} E^{-2.54} \text { particles } \mathrm{cm}^{-2} \mathrm{MeV}^{-1} \mathrm{~s}^{-1},
$$

which gives an integral flux above $100 \mathrm{MeV}$ of 1000 particles $\mathrm{cm}^{-2} \mathrm{~s}^{-1}$. 
A ten minute passage through the belt at such a flux level was used in computing the spallation in the worst possible case for the U.K.5 detector. Taking the mean path length of a proton in the satellite material and collimator as equivalent to $18 \mathrm{~g} \mathrm{~cm}^{-2}$ of CsI, allowance was made for energy loss to give a modified spectrum $j^{\prime}(E)$, which was used to compute the numbers of each isotope produced via

$$
N_{B}(Z, A)=600 \int_{100}^{2500} \frac{\sigma\left(A_{t}, E, Z, A\right)}{\sigma_{i}\left(A_{t}\right)}\left(1-e^{-t / \lambda}\right) \times \mathrm{G} . \mathrm{F} . \times j^{\prime}(E) \mathrm{d} E
$$

where

$$
t=\text { thickness of crystal, G.F.= geometric factor. }
$$

The lower limit of $100 \mathrm{MeV}$ was taken since below this energy the majority of particles lose their energy through ionisation without undergoing nuclear interactions.

There are very few inner belt protons above $2500 \mathrm{MeV}$ so that this upper limit was taken to restrict integration to a reasonable number of points. This calculation is thus based on a total dosage at the satellite of $6 \times 10^{5}$ protons $\mathrm{cm}^{-2}>100 \mathrm{MeV}$ for one passage through the S.A. Anomaly, resulting in $3 \times 10^{5}$ interactions in the central crystal. Throughout this paper, all background activity levels and spectra quoted for satellite conditions are based on this dosage and number of interactions. Results presented here can be simply scaled according to the dosage expected in any given satellite orbit.

Accurate integration of inner belt proton fluxes (Vette, 1966) over the satellite orbit mapped into B-L space (Dudziack et al., 1963) showed this dosage to be that expected for deepest anomaly penetration for a circular, $700 \mathrm{~km}, 32^{\circ}$ inclination orbit. Four successive orbits will receive approximately this dosage each day. The dosage to be expected in the best possible U.K.5 orbit (circular $500 \mathrm{~km}, 32^{\circ}$ inclination) is $1.6 \times 10^{5}$ particles $\mathrm{cm}^{-2}>100 \mathrm{MeV}$. This represents an improvement by a factor 4 on the numbers presented here.

\section{B. PRODUCTION BY A MONOENERGETIC BEAM AT $155 \mathrm{MeV}$}

A dosage giving $3 \times 10^{5}$ interactions was chosen for convenience of comparison with $A$.

\section{PRODUCTION BY COSMIC RAYS}

Because $\sigma\left(A_{t}, E, Z, A\right)$ is independent of energy for $E \geqslant 2.1 \mathrm{GeV}$, no integration was required. For a $2 \mathrm{GV}$ cut-off rigidity the integral flux of cosmic rays is 950 particles $\mathrm{m}^{-2} \mathrm{~s}^{-1} \mathrm{sr}^{-1}$ at solar sunspot maximum (Webber, 1967). This was calculated to give 0.55 interactions $\mathrm{s}^{-1}$ in the central crystal. Damage occurring for higher cut-off rigidities can simply be obtained by scaling these results according to the appropriate integral flux.

Tables I and II present these results for cases (A) to (C) for the predominant unstable isotopes used in predicting the spectrum for the case of $155 \mathrm{MeV}$ irradiation. All three cases are normalised to $3 \times 10^{5}$ nuclear interactions. Table I gives the short term decays used in predicting the spectra for times after irradiation up to one hour. 
TABLE I

Short term decays

\begin{tabular}{|c|c|c|c|c|c|}
\hline Isotope & Decay Mode and Energy (MeV) & $\begin{array}{l}\text { Half-Life } \\
\tau_{1 / 2} \text { (mins) }\end{array}$ & $\begin{array}{l}\text { Predicted } \\
\text { (A) } \\
\text { Inner Belt }\end{array}$ & $\begin{array}{l}\text { Nos. Prod } \\
\text { (B) } \\
155 \mathrm{MeV}\end{array}$ & $\begin{array}{l}\text { luced } \\
\text { (C) } \\
\text { Cosmic Rays }\end{array}$ \\
\hline${ }^{55} \mathrm{Cs}_{130}$ & $\begin{array}{ll}\beta^{+} & 1.97 \\
\beta^{-} & 0.442\end{array}$ & 30 & 2132 & 2122 & 475 \\
\hline${ }^{55} \mathrm{Cs}_{128}$ & $\begin{array}{ll}\beta^{+} \quad 3.0(70 \%) \\
& 2.5(30 \%) \\
\text { E.C. } & (25 \%) \\
\gamma & 0.460(20 \%) \\
& 0.285(20 \%)\end{array}$ & 3 & 5744 & 5632 & 2525 \\
\hline${ }^{55} \mathrm{Cs}_{127}$ & $\begin{array}{l}\text { E.C. } \\
\gamma \quad 0.406\end{array}$ & 360 & 7965 & 7460 & 4734 \\
\hline${ }^{55} \mathrm{Cs}_{126}$ & $\begin{array}{ll}\beta^{+} & 3.8(82 \%) \\
\text { E.C. }(18 \%) \\
\gamma \quad 0.386(38 \%)\end{array}$ & 1.6 & 9287 & 8105 & 7282 \\
\hline${ }^{54} \mathrm{Xe}_{125}$ & $\begin{array}{l}0.187 \\
0.056 \\
0.243 \\
\text { E.C. }\end{array}$ & 1080 & 2878 & 2287 & 2910 \\
\hline${ }^{54} \mathrm{Xe}_{123}$ & $\begin{array}{ll}\beta^{+} & 1.7 \\
\gamma & 0.148\end{array}$ & 120 & 4379 & 2712 & 6913 \\
\hline${ }^{53} \mathbf{I}_{123}$ & $\begin{array}{l}\text { E.C. } \\
\gamma \quad 0.160\end{array}$ & 780 & 8333 & 7929 & 3999 \\
\hline${ }^{53} I_{122}$ & 3.0 & 4 & 11923 & 10918 & 7796 \\
\hline${ }^{53} I_{121}$ & $\begin{array}{ll}\beta^{+} & 1.2 \\
\gamma & 0.21\end{array}$ & 96 & 14376 & 12345 & 12479 \\
\hline${ }^{52} \mathrm{Te}_{127^{\mathrm{a}}}$ & $\beta^{-} \quad 0.70$ & 560 & 1500 & 1500 & 1500 \\
\hline${ }^{51} \mathrm{Sb}_{118}^{\mathrm{m}}$ & 3.1 & 3.5 & 1573 & 967 & 2775 \\
\hline${ }^{51} \mathrm{Sb}_{117}$ & $\begin{array}{l}\text { E.C. } \\
y\end{array} 0.161$ & 168 & 2601 & 1376 & 5586 \\
\hline${ }^{51} \mathrm{Sb}_{116}$ & $\begin{array}{ll}\beta^{+} & 2.4 \\
\gamma & 1.3 \\
& 0.900\end{array}$ & 15 & 3579 & 1601 & 9204 \\
\hline${ }^{50} \mathrm{Sn}_{111}$ & E.C. $(71 \%)$ & 35 & 653 & 105 & 3427 \\
\hline
\end{tabular}

a Single Nucleon Removal.

E.C. Electron Capture, 
TABLE II

Long term decays

\begin{tabular}{|c|c|c|c|c|c|}
\hline Isotope & $\begin{array}{l}\text { Energies of } \gamma \text { rays }(\mathrm{MeV}) \\
\text { and branching ratio }\end{array}$ & $\begin{array}{l}\text { Half-Life } \\
\tau_{1 / 2} \text { (days) }\end{array}$ & $\begin{array}{l}\text { Predicted } \\
\text { (A) } \\
\text { Inner Belt }\end{array}$ & $\begin{array}{l}\text { Nos. Prod } \\
\text { (B) } \\
\text { t } 155 \mathrm{MeV}\end{array}$ & $\begin{array}{l}\text { duced } \\
\text { (C) } \\
\text { Cosmic Rays }\end{array}$ \\
\hline${ }^{55} \mathrm{Cs}_{132}{ }^{\mathrm{a}}$ & E.C. 0.670 & 6.2 & 1500 & 1500 & 1500 \\
\hline${ }^{55} \mathrm{Cs}_{131}$ & E.C. & 10 & 1145 & 1106 & 175 \\
\hline${ }^{55} \mathrm{Cs}_{129}$ & E.C. 0.380 & 1.3 & 3669 & 3672 & 1163 \\
\hline${ }^{55} \mathrm{Cs}_{127}$ & E.C. $0.406(80 \%)$ & 0.25 & 7965 & 7460 & 4734 \\
\hline${ }^{54} \mathrm{Xe}_{131}^{\mathrm{mm}}$ & 0.163 & 12 & 46 & 45 & 7 \\
\hline${ }^{54} \mathrm{Xe}_{129}^{\mathrm{m}}$ & $0.196 ; 0.040$ & 8 & 244 & 244 & 77 \\
\hline${ }^{54} \mathrm{Xe}_{127}$ & E.C. $0.370(40 \%) ; 0.203(60 \%)$ & 34 & 1002 & 938 & 596 \\
\hline${ }^{54} \mathrm{Xe}_{125}$ & E.C. $0.187 ; 0.243$ & 0.7 & 2878 & 2287 & 2909 \\
\hline${ }^{54} \mathrm{Xe}_{123}$ & 0.148 & 0.08 & 4379 & 2712 & 6913 \\
\hline${ }^{53} \mathbf{I}_{126} \mathrm{a}$ & E.C. $(55 \%) 0.386(34 \%) ; 0.650(33 \%)$ & 13 & 1500 & 1500 & 1500 \\
\hline${ }^{53} \mathbf{I}_{125}$ & E.C. & 60 & 2898 & 2750 & 692 \\
\hline${ }^{53} \mathbf{I}_{124}$ & E.C. $(70 \%) 0.605(95 \%)$ & 4 & 5157 & 4964 & 1769 \\
\hline${ }^{53} \mathbf{I}_{123}$ & E.C. 0.160 & 0.5 & 8333 & 7929 & 3999 \\
\hline${ }^{52} \mathrm{Te}_{125}^{\mathrm{m}}$ & $0.110 ; 0.035$ & 58 & 136 & 129 & 32 \\
\hline${ }^{52} \mathrm{Te}_{123}^{\mathrm{m}}$ & $0.089 ; 0.159$ & 104 & 685 & 652 & 328 \\
\hline${ }^{52} \mathrm{Te}_{121}^{m}$ & $0.082 ; 0.214$ & 154 & 2560 & 2199 & 2222 \\
\hline${ }^{52} \mathrm{Te}_{121} \mathrm{~b}$ & $0.570(87 \%) ; 0.506(13 \%)$ & 17 & 14376 & 12345 & 12479 \\
\hline${ }^{52} \mathrm{Te}_{119}$ & E.C. & 4.5 & 6070 & 4264 & 8638 \\
\hline${ }^{51} \mathrm{Sb}_{122}$ & $0.566(66 \%)$ & 2.8 & 71 & 65 & 46 \\
\hline${ }^{51} \mathrm{Sb}_{120}$ & E.C. $0.089 ; 0.199$ & 6 & 397 & 312 & 446 \\
\hline${ }^{5} \mathrm{Sb}_{119}$ & E.C. & 1.66 & 832 & 585 & 1184 \\
\hline${ }^{50} \mathrm{Sn}_{117}^{\mathrm{m}}$ & $0.159 ; 0.162$ & 14 & 283 & 150 & 608 \\
\hline${ }^{50} \mathrm{Sn}_{113}$ & E.C. 0.392 & 120 & 2249 & 562 & 9162 \\
\hline${ }^{49} \mathrm{Tn}_{111}$ & $0.172 ; 0.247$ & 2.8 & 1318 & 212 & 6920 \\
\hline
\end{tabular}

a Single Nucleon Removal.

b Secondary Product.

E.C. Electron Capture.

Table II gives the long term decays used for predicting the spectra up until 68 days.

Figure 2 gives the mass yield curves for the three cases. The similarity of the spallation predicted in situations (A) and (B) can be clearly seen. In the case of cosmic ray induced spallation, the relatively greater importance of low $(Z, A)$ products may be noticed.

Figure 3 compares production rates for cases (A) and (B) down to $Z=49$. It should 


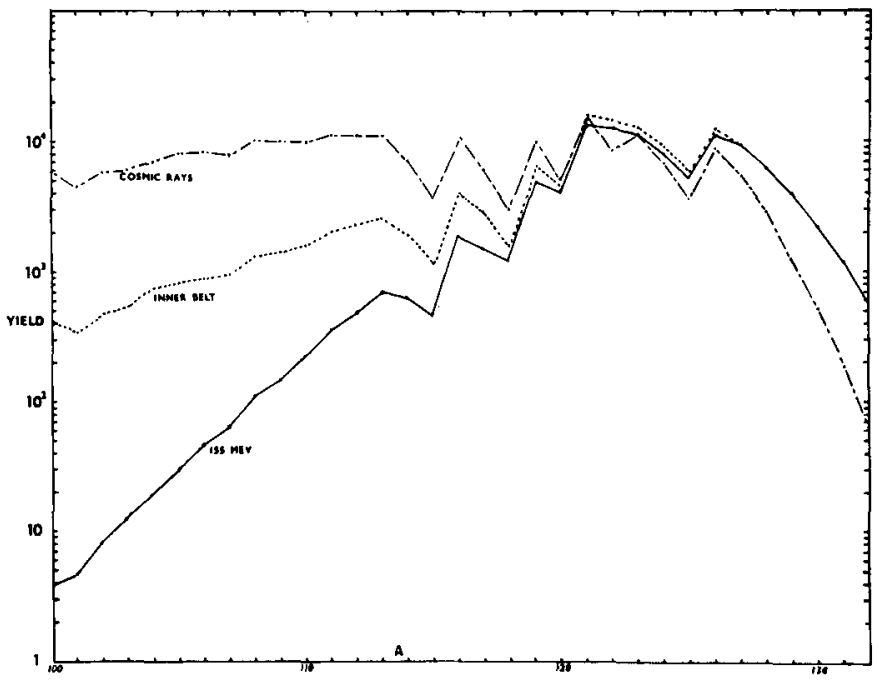

Fig. 2. Mass yield curves predicted by the Rudstam formula for proton induced spallation in CsI, given down to atomic mass number (A) 100 and normalised to $3 \times 10^{5}$ interactions.

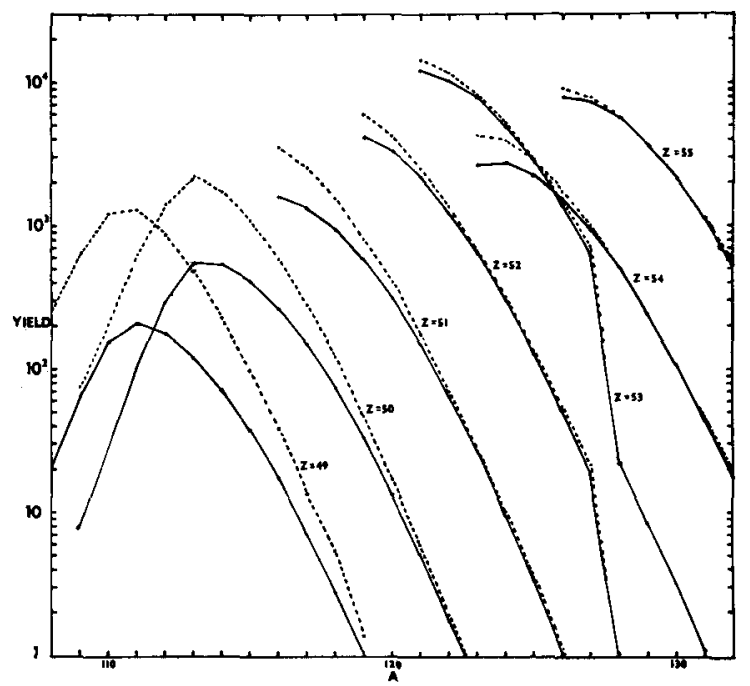

Fig. 3. The yield of isotopes of $Z>49$ as predicted by the Rudstam formula for $3 \times 10^{5}$ proton interactions in CsI. Inner belt produced spallation products are shown by a dotted line, $155 \mathrm{MeV}$ produced spallation products by a solid line.

be noted that throughout this work the total number of interactions is calculated using the total geometrical cross-section. The total number of spallation products predicted from the formula can be less than this so that the curves of Figures 2 and 3 are not normalised to contain the same area. For curves (A) and (B) the areas contained are sufficiently similar to justify direct scaling for predicting inner belt induced activity from results at $155 \mathrm{MeV}$, 


\section{Nuclide Production by Neutron Interactions}

Both albedo neutrons from the earth's atmosphere and neutrons produced throughout the spacecraft as secondaries in spallation interactions can contribute to damage in the central crystal. For low energy neutrons $(<1 \mathrm{MeV})$ both $\mathrm{Cs}$ and I have appreciable capture cross-sections. Thermal neutron capture by $\mathrm{I}^{127}$ has a cross-section of $6.3 \mathrm{~b}$ and yields the unstable isotope $\mathrm{I}^{128}\left(\tau_{1 / 2}=25 \mathrm{~min}, \beta^{-} 2.12 \mathrm{MeV}(76 \%), 1.67 \mathrm{MeV}\right.$ $(16 \%) ; \gamma 0.46 \mathrm{MeV}(17 \%))$. The equivalent cross-section for $\mathrm{Cs}^{133}$ is $29 \mathrm{~b}$ but this yields $\mathrm{Cs}^{134}$ of half-life 2 years, and several years would be required to build up an equilibrium decay rate.

High energy neutrons will contribute to spallation damage (the Rudstam formula is unchanged if the incident particle is a neutron).

The case of capture of secondaries has been considered by Peterson (1965) as the source of the observed activity in NaI. The problem of thermalising the neutrons in sufficient quantities was not resolved.

We here attempt to obtain an estimate of the relative importance of this process compared with direct proton interactions by considering the work of Ebeoglu et al. (1966) on nuclear cascades in iron meteorites. These workers calculate the relative reaction rates of primaries and secondaries as a function of depth for a $400 \mathrm{MeV}$ proton beam incident on an iron slab. At a depth of $4 \mathrm{~cm}\left(31 \mathrm{~g} \mathrm{~cm}^{-2}\right)$ this ratio is calculated to be $2: 1$. For this depth an energy spectrum of secondaries is also given from which it is possible to deduce that less than $1 \%$ of these secondaries have energies sufficiently low $(<1 \mathrm{MeV})$ to undergo capture. Hence the contribution of $\mathrm{I}^{128}$ from this source will be of the same order of magnitude as any one of the many other isotopes considered previously and will not much alter the predicted spectrum. The majority of these secondaries will add to spallation damage. An increase by a factor 1.5 in the total number of particles is predicted for this depth.

Inner belt protons reaching the central crystal have a lower characteristic energy and on average traverse less material $\left(18 \mathrm{~g} \mathrm{~cm}^{-2}\right)$ so that the above figures represent a considerable overestimate of the importance of secondaries.

An atmospheric neutron leakage flux spectrum as a function of latitude has been predicted by Lingenfelter (1963). At solar sunspot maximum and $40^{\circ}$ latitude the predicted fluxes are $\left(\mathrm{cm}^{-2} \mathrm{~s}^{-1}\right)$ :

$$
\begin{array}{lll}
<1 \mathrm{MeV} & 1 \text { to } 10 \mathrm{MeV} & >10 \mathrm{MeV} \\
0.190 & 0.091 & 0.017 .
\end{array}
$$

The contribution of high energy albedo neutrons to spallation is clearly negligible in comparison with cosmic ray damage (flux $\simeq 0.6 \mathrm{~cm}^{-2} \mathrm{~s}^{-1}$ at the satellite).

The relative importance of capture of low energy albedo neutrons was calculated by integration over the low energy portion of the spectrum, making due allowance for absorption in $18 \mathrm{~g} \mathrm{~cm}^{-2}$ of satellite material. This calculation showed that for a $32^{\circ}$ orbit, $\mathrm{I}^{128}$ production from such a source would occur at approximately one third the total rate of radioactive nuclide production by cosmic ray induced spallation. In an 
equatorial orbit the albedo fluxes would be reduced by a factor three, whilst for cosmic ray fluxes the reduction would be by a factor of ten. Capture of albedo neutrons to yield $\mathrm{I}^{128}$ would thus seem capable of providing a background comparable with that resulting from cosmic ray induced activity.

\section{Results of the Proton Beam Experiment}

\section{A. EXPERIMENTAL PROCEDURE}

A cylindrical CsI crystal ( $5 \mathrm{~cm}$ long by $3.4 \mathrm{~cm}$ diameter), similar to the central crystal of the U.K.5 X-ray telescope, was employed. The crystal was exposed to a beam of $155 \mathrm{MeV}$ protons provided by the synchrocyclotron at AERE, Harwell. The integrated dosage given to the crystal was monitored to $25 \%$ accuracy using an ionization chamber. Two exposures using different dosages were effected in order to observe both short and long term behaviour. On the first occasion the crystal was given a dosage of $4 \times 10^{8}$ protons over three minutes. Taking the interaction length, $\lambda$, for inelastic collisions in CsI as $38 \mathrm{~cm}$, this gives $5 \times 10^{7}$ interactions. On the second occasion the dosage was $1.2 \times 10^{10}$ protons over one minute, giving $9.6 \times 10^{8}$ interactions. The light pulses resulting from radioactive decays inside the irradiated crystal were examined by a photomultiplier, pulse-height analyser system previously calibrated using standard X-ray sources. The energy spectra measured with the P.H.A. were plotted out at regular intervals in order to observe the decay characteristics. Various P.H.A. gain values were used in order to observe different parts of the spectrum. The first experiment gave accurate information over the first two days after irradiation, after which time laboratory background became important. The second experiment saturated the electronics over the first hour but gave information on the spectral shape up until 105 days before other sources of background became dominant.

\section{B. EXPERIMENTAL RESULTS COMPARED WITH THEORETICAL PREDICTION OF SPECTRA}

Information on the decay schemes of the spalled nuclei was obtained from Strominger, Hollander and Seaborg (1958) and used to calculate decay rates as a function of time after irradiation. Use was made of the response of the crystal to standard X-ray sources and of Monte Carlo calculations of the photopeak efficiency and Compton plateau level to estimate the spectra for the predicted isotope decays. The predicted spectra were compared with experimental results at different times after irradiation. The $\beta^{+}$contributions were assumed to have a maximum at half of the maximum energy available (maximum energy of $\beta^{+}+$annihilation energy).

Two major difficulties in the prediction process are the lack of complete information on certain decay schemes and the uncertainty as to whether or not certain spalled nuclei would be produced in the metastable condition. The results of our predictions are compared with experiment in Figures 4 and 5. Figure 4 compares the prediction of short term behaviour obtained using Table I with the experimental results of the first irradiation over the energy range $60 \mathrm{keV}$ to $2.4 \mathrm{MeV}$. General agreement to better than a factor of three in the absolute intensity and $\pm 50 \mathrm{keV}$ in the position of peaks is obtained. A short-lived peak at $390 \mathrm{keV}$ is predicted but not observed, and experiment 


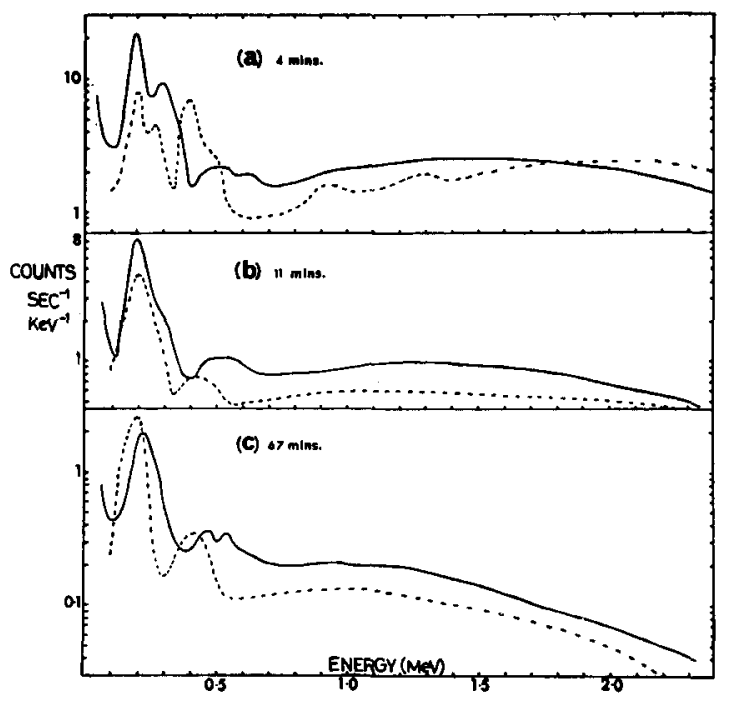

Fig. 4. Experimentally observed spectra (shown solid) obtained from the first irradiation $\left(5 \times 10^{7}\right.$ interactions), compared with predictions (shown dotted) based on the Rudstam formula and the Table of Isotopes, for (a) $4 \mathrm{~min}$, (b) $11 \mathrm{~min}$, (c) $67 \mathrm{~min}$ after irradiation.

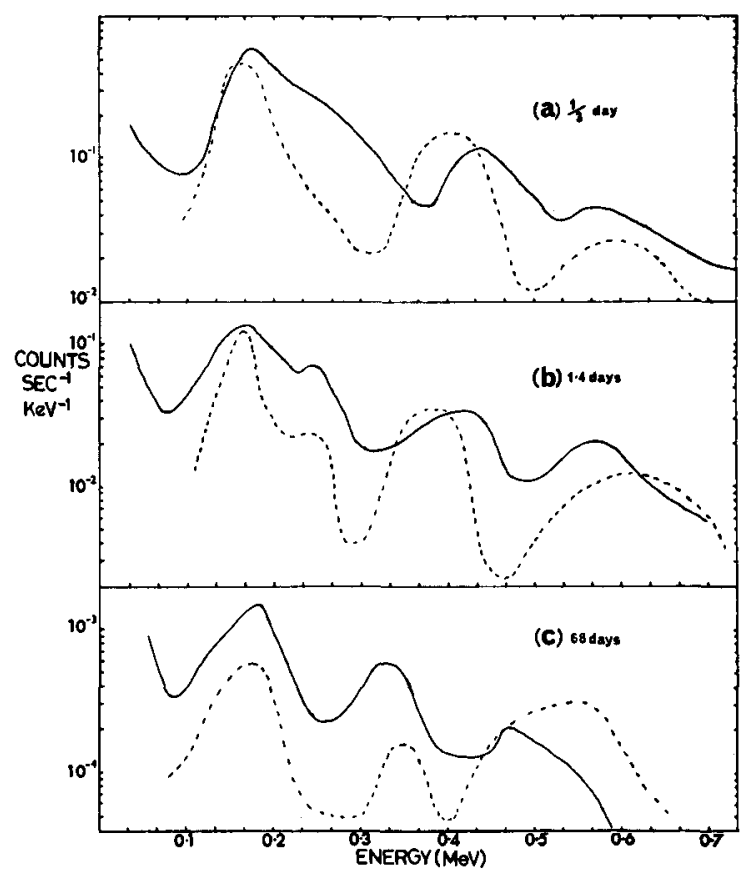

Fig. 5. Experimentally observed spectra (shown solid) from the second irradiation $\left(9.6 \times 10^{8}\right.$ interactions) compared with predictions (shown dotted) based on the Rudstam formula and the Table of Isotopes, for (a) $\frac{f}{3}$ day, (b) 1.4 days, (c) 68 days after irradiation. 
would seem to show an annihilation line at $510 \mathrm{keV}$ which is not predicted. An order of magnitude estimate indicates that the latter line could result from the escape of $\beta^{+}$ particles into the surrounding lead shield with re-entry of some of the annihilation $\mathrm{X}$-rays into the crystal.

Figure 5 compares the long-term behaviour predicted from Table II with some results of the 2 nd irradiation for energies from $60 \mathrm{keV}$ to $700 \mathrm{keV}$. Better than order of magnitude agreement over the entire range of the experiment ( $4 \mathrm{~min}$ to 68 days)can thus be seen. Activity levels between peaks are necessarily underestimated by this process as they are a superposition of a large number of decays not taken into account.

These comparisons take no account of structure below $60 \mathrm{keV}$. As can be seen from Figure 7, this comprises a single peak at about $40 \mathrm{keV}$. This peak can be well explained as a superposition of all electron capture decays resulting in emission of X-ray quanta equal in energy to the $\mathrm{K}$ shell electron. These energies range from $35.9 \mathrm{keV}$ for $\mathrm{Cs}$ isotopes to $29.2 \mathrm{keV}$ for $\mathrm{Sn}$ isotopes. From Table I this would predict a level of $0.02 \mathrm{keV}^{-1} \mathrm{~s}^{-1}$ after $1 \mathrm{hr}$ on the scale of Figure 7, which compares favourably with $0.05 \mathrm{keV}^{-1} \mathrm{~s}^{-1}$ observed experimentally.

These results would indicate that the nature of the activating process is well understood in terms of spallation and that the Rudstam formula used in conjunction with radiochemical data can yield better than order of magnitude predictions.

\section{Prediction of Radioactivity Induced on Board Satellite}

\section{A. INNER BELT}

Thus far it has been shown that the Rudstam formula gives a good explanation of the activity caused by $155 \mathrm{MeV}$ protons and that the formula predicts a close similarity between the spallation produced at this energy and that due to an inner belt spectrum. Hence scaling the experimental results by the appropriate dosage should produce a good estimate of the activity caused by passage through the South Atlantic Anomaly.

Figures 6 and 7 present the experimental results scaled to the dosage $\left(6 \times 10^{5}\right.$ protons $\mathrm{cm}^{-2}$ ) used in the theoretical prediction of nuclide production for the worst case of S.A. Anomaly traversal. Figure 6 gives information over the energy range from $50 \mathrm{keV}$ to $2.3 \mathrm{MeV}$. Figure 7 gives the details of the low energy part of the spectra from $20 \mathrm{keV}$ to $500 \mathrm{keV}$.

The long-term behaviour can be obtained from the experimental curves of Figure 5 by scaling by the factor $3.1 \times 10^{-4}$. Figure 6 also includes the expected background counting rate spectrum due to the diffuse cosmic X-ray background (both entry through the telescope aperture and leakage through the collimator, as predicted by a Monte Carlo technique, have been considered). It can be seen that the activity takes over $2 \mathrm{hr}$ to fall to a comparable level.

Figure 8 shows how the counting rate would die away as a function of time for certain of the energy channels that are to be employed in the U.K.5 experiment. The effect of superposing a large number of half-lives is clearly shown.

In practice the satellite will make several successive passages through the S.A. 


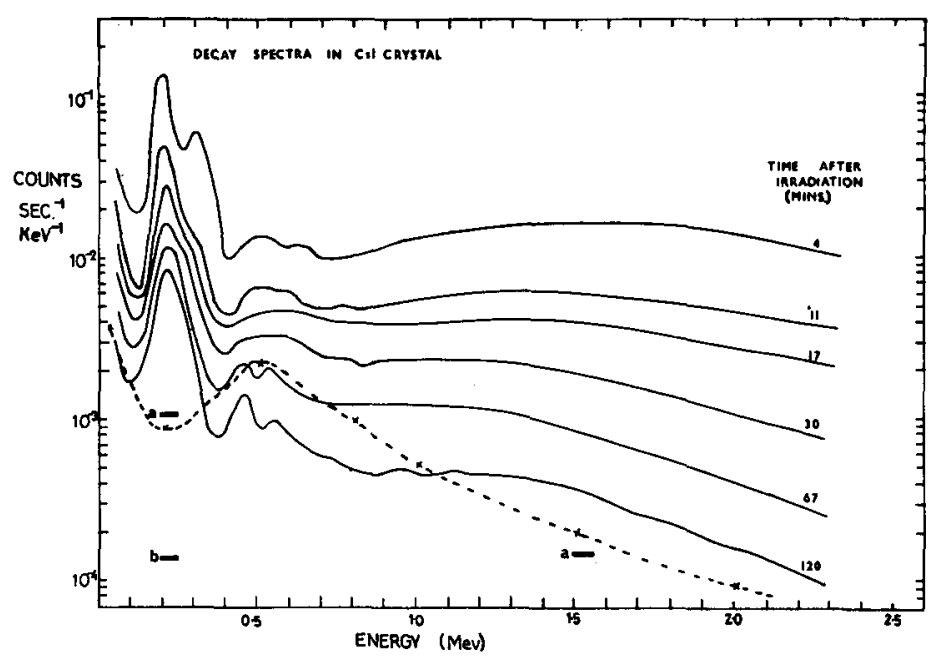

Fig. 6. Predicted spectra (shown solid) for worst case inner belt traversal, obtained by scaling experimentally observed spectra to $3 \times 10^{5}$ interactions, presented for times up to $2 \mathrm{hr}$ after irradiation. The dotted curve gives the estimated background due to the diffuse cosmic X-ray flux (both entry through aperture and leakage through the collimator). ' $a$ ' gives the expected level of background due to cosmic-ray damage for a $2 \mathrm{GV}$ cut-off, and ' $b$ ' the same for a $14.9 \mathrm{GV}$ cut-off.

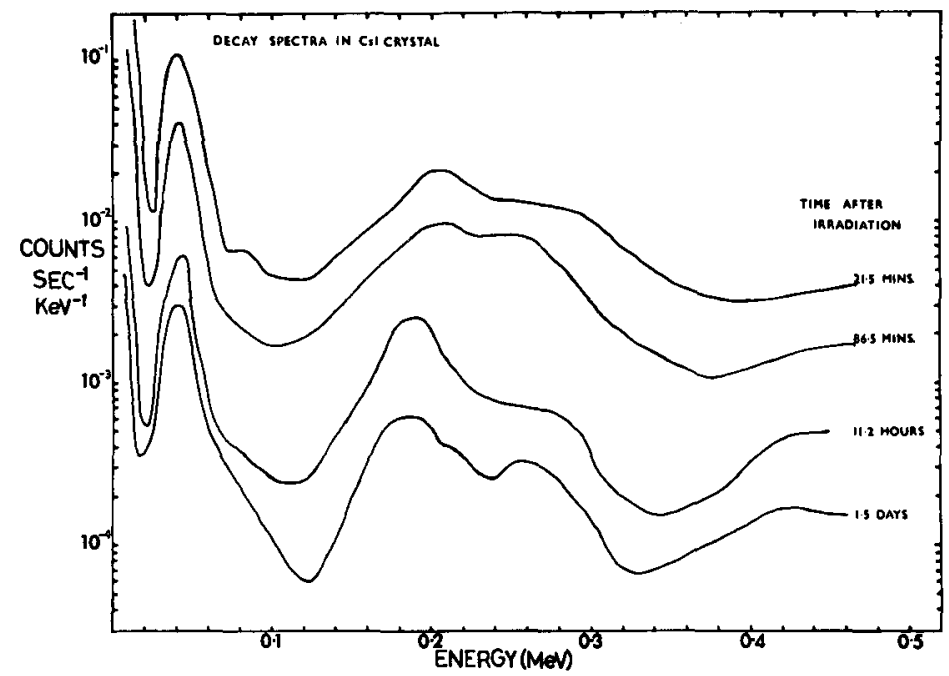

Fig. 7. Predicted spectra due to inner belt spallation for the low energy $\mathrm{X}$-ray region.

Anomaly and will then spend a number of orbits when there is no such irradiation. It is to be expected that there will be a build-up of background counting rate throughout the history of the satellite due to the long half-life isotopes. A worst case estimate of this effect has been made for the 200 to $266 \mathrm{keV}$ channel which shows a particularly well marked peak in both short and long term behaviour. It was decided to use a simple model of four successive dosages at the above level at $100 \mathrm{~min}$ intervals followed by a 


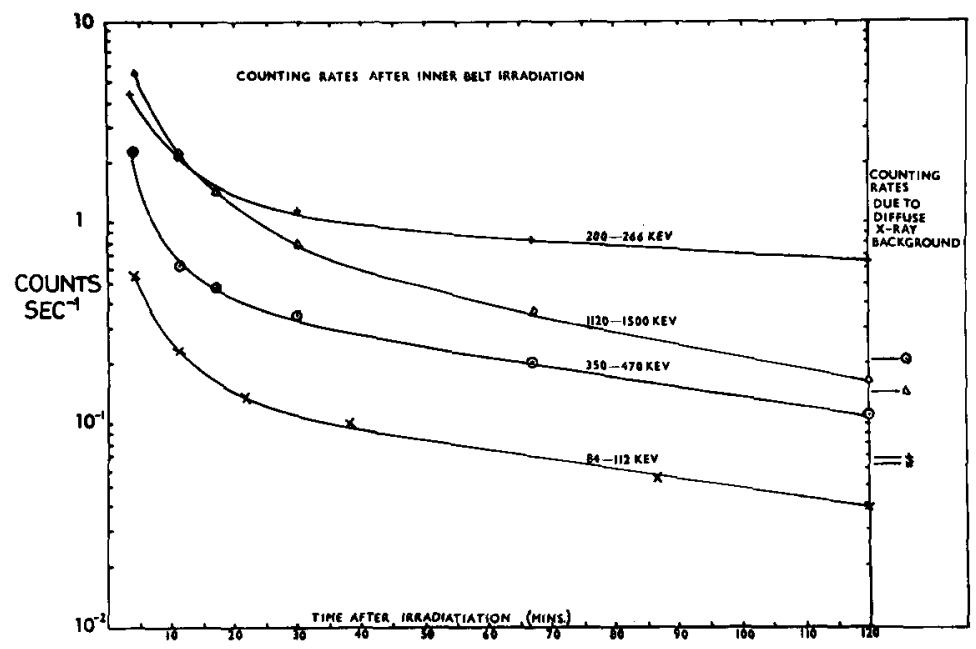

Fig. 8. Predicted counting rates as a function of time after inner belt irradiation for four energy channels. The counting rates in the same channels, due to the diffuse cosmic X-ray flux, are presented at the right hand side.

day without irradiation. These results predict that the background rate in this channel at a half day after the latest irradiation would increase from 0.43 count s$^{-1}$ on the first day to 0.80 count s$^{-1}$ after 10 days, to 1.0 count s $^{-1}$ after 60 days, and to 1.4 counts $\mathrm{s}^{-1}$ after 1 year. Such a build-up of background is capable of keeping the level of background due to induced radioactivity above that due to the diffuse cosmic X-ray background (see Figure 6).

The predicted level of activity in the crystal is $0.25 \mathrm{~g}^{-1} \mathrm{~s}^{-1}$ immediately after irradiation. For a $550 \mathrm{~km}, 33^{\circ}$ inclination orbit the orbital integration of proton fluxes reduces this level to $0.07 \mathrm{~g}^{-1} \mathrm{~s}^{-1}$, which compares remarkably well with a typical value of $0.08 \mathrm{~g}^{-1} \mathrm{~s}^{-1}$ reported by Peterson (1965) for a NaI crystal in such an orbit.

It should be noted that these results were obtained for a single crystal of CsI. In the case of an active collimator the high energy part of the spectrum would be somewhat reduced in intensity as partial energy losses in the central crystal can produce anticoincidence pulses in the collimator. With the U.K.5 arrangement it is estimated that the high energy end ( 1 to $2 \mathrm{MeV}$ ) would be decreased by about $50 \%$ in this way.

\section{B. COSMIC RAYS}

In addition to these intermittent passages through the S.A. Anomaly, the satellite will be subject to a flux of cosmic rays that varies with the magnetic latitude. If the flux were constant, an equilibrium situation would be reached when the decay rate of each isotope would equal its production rate. A worst case estimate of the cosmic ray induced background can be obtained by considering a constant flux appropriate to the highest magnetic latitude that the satellite attains. This was taken to give a $2 \mathrm{GV}$ rigidity cut-off.

As has been seen above, the spectrum to be expected will differ considerably from that due to inner belt irradiation. However a comparison of activity levels can be made 
by considering the peaks at around $200 \mathrm{keV}$ and $1.5 \mathrm{MeV}$. The predicted levels of these peaks for a $2 \mathrm{GV}$ cut-off are marked in on Figure 6, as are the levels predicted for an equatorial orbit at $14.9 \mathrm{GV}$ cut-off. These levels are presented for solar sunspot maximum. At sunspot minimum the $2 \mathrm{GV}$ levels would be increased by a factor 1.6 and the 14.9 GV levels by 1.1 .

\section{Implications of these Results for the Interpretation of the Diffuse Cosmic X-Ray Spectrum}

Whereas measurements of discrete X-ray sources can be readily corrected for every kind of background, accurate determination of the isotropic component of cosmic $X$-ray radiation involves a subtraction of every conceivable form of background, for example charged particle counts, induced radioactivity, atmospheric X-rays and solar $\mathrm{X}$-ray bursts. Of these sources of background, induced radioactivity is the most difficult to allow for, as it can never be totally eliminated from the data by careful detector design or data selection, and adequate correction requires knowledge of the spectrum as a function of time.

Inspection of Figures 6 and 7 shows that major features of our results are the well marked peaks at $40 \mathrm{keV}$ and $200 \mathrm{keV}$, and a flat spectrum above $1 \mathrm{MeV}$. The $40 \mathrm{keV}$ peak is a superposition of $\mathrm{K}$ capture decays, and the spectrum above $1 \mathrm{MeV}$ results from numerous $\beta^{+}$decays. The $200 \mathrm{keV}$ peak is a superposition of several isotopes (e.g. ${ }^{54} \mathrm{Xe}_{125},{ }^{53} \mathrm{I}_{123},{ }^{53} \mathrm{I}_{121},{ }^{51} \mathrm{Sb}_{117}$ ). Tables I and II indicate that these features will also occur in the cosmic ray induced activity. In interplanetary space the cosmic ray produced background exceeds the diffuse X-ray flux (extrapolated as a $E^{-2}$ law) at energies greater than $1 \mathrm{MeV}$. This applies whatever the detector design, as both cosmic X-ray counts and radioactivity counts increase in proportion to the mass of scintillator material. Application of the Rudstam formula calculation to the case of $\mathrm{NaI}$ shows that these features will again be present and that the activity will be the same as that for CsI to within a factor of 2. Cosmic X-ray spectra above $20 \mathrm{keV}$ are usually measured using alkali halide crystals. Measurements by various workers on the diffuse component spectrum have been summarised (Peterson, 1970), and the results are presented in Figure 9. There is evidence of structure at the above-mentioned energies, which could result from cosmic ray or inner belt produced background activity.

\section{Conclusions}

Direct interaction of inner belt protons in the central crystal of the U.K.5 detector will provide the dominant source of background for the proposed orbit. A good case for equatorial orbits for such experiments is thus made.

A good understanding of this background has been provided in terms of spallation interactions and subsequent decay of unstable isotopes. The Rudstam formula, used in conjunction with radiochemical data, would seem capable of yielding better than order of magnitude predictions of such activity. A general method for predicting activity in satellite-borne material has thus been provided. 


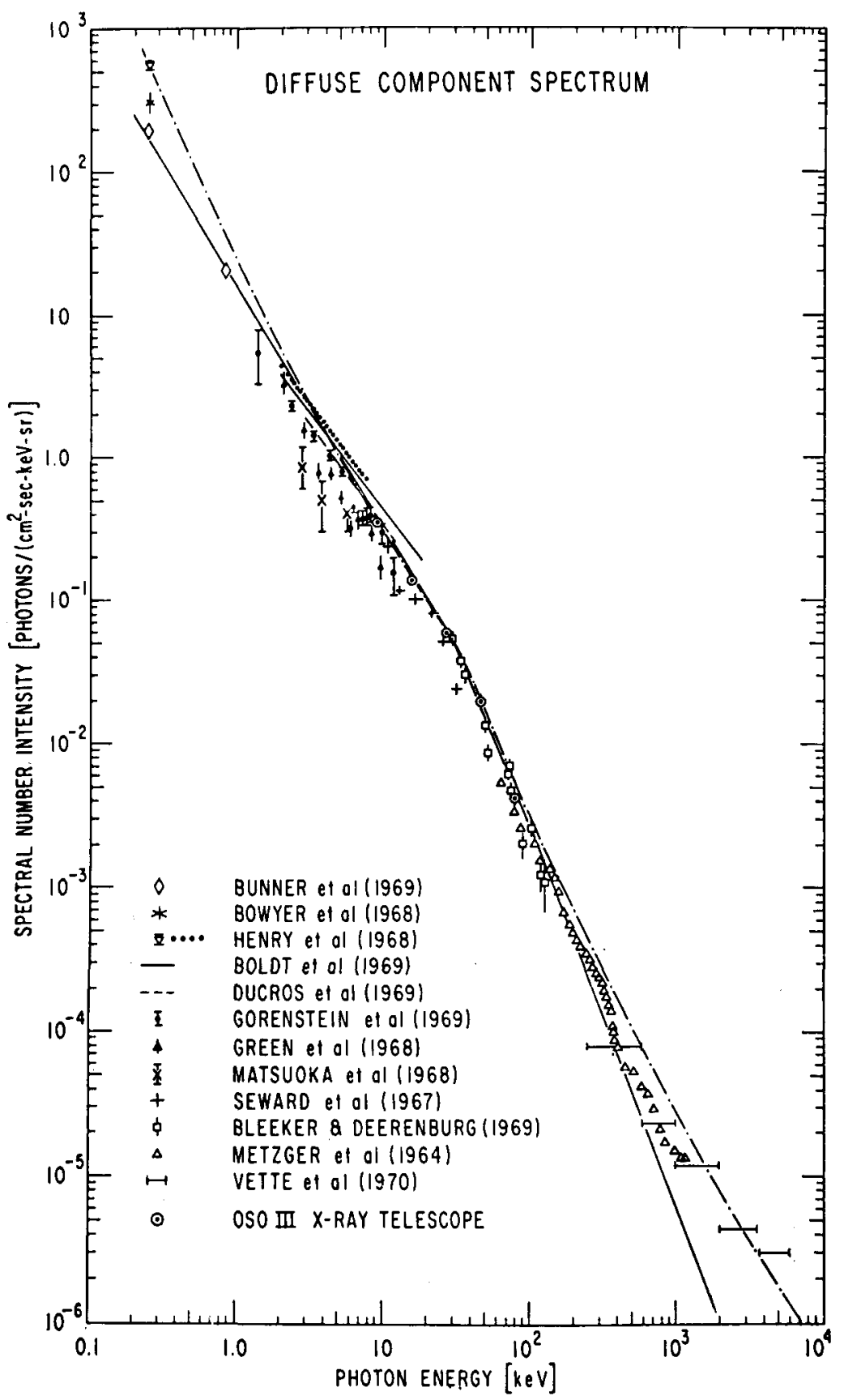

Fig. 9. The diffuse component spectrum of cosmic X-rays. Results summarised by Peterson (1970). The results show a break in the spectrum at $40 \mathrm{keV}$ and fiattening in the region $>1 \mathrm{MeV}$. There is also some evidence of structure in the region around $200 \mathrm{keV}$. 
In view of the experimentally observed peaks and the flatness of the spectrum in the high energy region, it is suggested that particular care be taken in interpreting the observed spectra of cosmic X-ray sources. In particular, breaks in the spectra at $40 \mathrm{keV}$, $200 \mathrm{keV}$ and $1 \mathrm{MeV}$ could result from an inadequate allowance for background.

\section{Acknowledgements}

We are indebted to Dr I. Blair and other members of the synchrocyclotron group at AERE Harwell for their help in providing and monitoring the proton beam.

We gratefully acknowledge a number of fruitful discussions with Dr J. J. Quenby and Dr A. R. Engel.

Thanks are due to Dr A. R. Engel and Mr D. J. Damaske for their help in performing the experiment.

Both authors would like to acknowledge their S.R.C. studentships held during this work.

Note added in proof. Reference to a more modern Table of Isotopes (6th Edition, 1967, by Lederer, C. M., Hollander, J. M., and Perlman, I., published by John Wiley and Sons) has shown that the $\gamma$-ray decay of ${ }^{55} \mathrm{Cs}_{126}$ will be detected as a $0.426 \mathrm{MeV}$ line with a branching ratio of $7 \%$ rather than $38 \%$ (see Table I). This correction removes the predicted but unobserved peak at $390 \mathrm{keV}$ in Figure 4(a).

\section{References}

Dennis, B. R.: 1970, Private Communication,

Dudziak, W. F., Kleinecke, D. D., and Kostigen, T. J.: 1963, 'Graphic Displays of Geomagnetic Geometry', RM 63 TMP-2, DASA 1372, General Electric Company.

Ebeoglu, D. B., Wainio, K. M., More, K., and Tiffany, O. L.: 1966, 'Monte Carlo Calculations of Radionuclide Production in Iron Targets bombarded with $400 \mathrm{MeV}$ Protons', J. Geophys. Res. 71, 1445.

Elliot, H.: 1965, 'Some Cosmic Ray and Radiation Belt Observations based on Data from the Anton 302 G.M. Counter in Atiel I', in Radiation trapped in the Earth's Magnetic Field (ed. by B. M. McCormac), D. Reidel Publishing Company, Dordrecht-Holland, p. 76.

Funk, H., and Rowe, M. W.: 1967, 'Spallation yield of Xenon from $730 \mathrm{MeV}$ Proton Irradiation of Barium', Earth Planet. Sci. Letters 2, 215.

Lingenfelter, R. E.: 1963, 'The Cosmic Ray Neutron Leakage Flux', J. Geophys. Res. 68, 5633.

Peterson, L. E.: 1965, 'Radioactivity induced in Sodium Iodide by Trapped Protons', J. Geophys. Res. 70, 1762.

Peterson, L. E.: 1970, 'An SKY survey Experiment for 0.3-10 MeV $\gamma$-Ray sources for HEAO-A', U.C.S.D., 3674.

Rudstam, G.: 1966, 'Systematics of Spallation Yields', Z. Naturforsch. 21a, 1027.

Schwartz, D. A.: 1969, 'The Spatial Distribution of the Diffuse Component of Cosmic X-rays', Thesis, U.C.S.D.

Strominger, D., Hollander, J. M., and Seaborg, G. T.: 1958, 'Table of Isotopes', Rev. Mod. Phys. 30, 585.

Vette, J. I.: 1966, 'Models of the Trapped Radiation Environment, Volume I: Inner Zone Protons and Electrons', NASA SP-3024.

Waddington, C. J.: 1969, 'The Fragmentation of Cosmic Ray Nuclei in Interstellar Hydrogen', Univ. of Minnesota Preprint.

Webber, W. R.: 1967, 'The Spectrum and Charge Composition of the Primary Cosmic Radiation', Handbuch der Physik, 46, 181. 